\title{
Ni chair ni poisson : tryphon-le-coupé
}

\section{Assia Popova}

\section{(2) OpenEdition}

Journals

Édition électronique

URL : https://journals.openedition.org/clo/671

DOI : $10.4000 /$ clo. 671

ISSN : 2266-1816

Éditeur

INALCO

\section{Édition imprimée}

Date de publication : 1 janvier 2009

Pagination : 107-145

ISBN : 978-2-85831-188-0

ISSN : 0396-891X

\section{Référence électronique}

Assia Popova, « Ni chair ni poisson : tryphon-le-coupé », Cahiers de littérature orale [En ligne], 66 | 2009, mis en ligne le 25 février 2013, consulté le 01 juillet 2021. URL : http://journals.openedition.org/clo/ 671 ; DOI : https://doi.org/10.4000/clo.671

Ce document a été généré automatiquement le 1 juillet 2021.

\section{(c) (†) 8)}

Cahiers de littérature orale est mis à disposition selon les termes de la Licence Creative Commons Attribution - Pas d'Utilisation Commerciale 4.0 International. 


\title{
Ni chair ni poisson : tryphon-le- coupé
}

\author{
Assia Popova
}

\section{NOTE DE L'ÉDITEUR}

Article paru pour la première fois dans Cahiers de littérature orale, $\mathrm{n}^{\circ}$ 3, 1977, p. 15-69.

\section{LE DÉMON DES VIgNES}

1 Les pères de l'Église orthodoxe qui sous le nom de saint Tryphon ont sacralisé le daimon des vignes, des arbres fruitiers et des loups, ont été non seulement de bons connaisseurs des traditions populaires des Balkans, mais aussi des sémanticiens astucieux. En effet, le vocable $\tau \rho v ́ \varphi \omega v$ qui signifie "dissolu », " efféminé », apparaît comme une excellente désignation pour son homologue «chrétien»-la divinité antique aux mœurs et agissements suspects.

2 Dans la tradition bulgare le saint patron des vignerons, des maraîchers et des taverniers, représenté sur les icônes comme un personnage camus tenant un koser (serpe à tailler), se trouve affublé des sobriquets de l'Ivrogne, le Camard et surtout le Coupé (Pijanica, Čipija, Zarezan).

3 À partir de son nom subversif - Tryphon ${ }^{1}$ - les Bulgares ont forgé le verbe «tryphoniser» (trifonisvam) qui veut dire: «tailler rituellement les vignes» et "menacer de couper ou battre les arbres et les femmes stériles à la Saint-Tryphon ».

4 Le terme liturgique "tryphoniser ", ainsi que la représentation iconographique de Tryphon Zarezan, on s'en doute, se réfèrent aux pratiques rituelles.

5 Ainsi, le $1^{\text {er }}$ a.s. $/ 14$ n.s. février ${ }^{2}$, les hommes en joyeuse procession se rendent dans les vignes où chaque propriétaire coupe trois sarments de trois (ou quatre) ceps différents de son vignoble : au centre et à deux extrémités (ou bien dans les quatre coins). Sur 
l'endroit de la coupure le tailleur met une pincée de suie ${ }^{3}$ en appuyant avec les doigts. Si la suie se mouille, c'est un bon signe, car cela signifie que la sève monte; sinon les vignes sont gelées. Chaque fois, après avoir taillé le cep, le vigneron l'arrose avec du vin, des cendres et parfois avec de l'eau bénite. Le «sang de saint Tryphon », c'est-àdire le vin apporté par le tailleur dans une gourde de sa maison, est destiné à fortifier les vignes et à donner une belle couleur et un bon goût à la future récolte.

Après la cérémonie de la taille, on procède à l'élection d'un nouveau roi des vignes que l'on appelle souvent "tryphon " et son règne dure de un à trois ans, "tryphonie " (trifunstvo). Cette élection se passe sur le lieu de sacrifice (obročište), dans les vignes, pendant le repas commun sous (ou près de) l'arbre sacré - un chêne, orme ou bouleau'. Le menu de ce festin joyeux et solennel se compose d'un veau bouilli ou d'un mouton rôti qui sera tué et cuisiné sur place, et des provisions apportées par chaque participant : un poulet au riz, un pain rituel décoré avec des vignes et des grappes de raisin moulées, et aussi parfois de la banica (pâte feuilletée au fromage et aux œufs). Tout ceci est abondamment arrosé avec du vin et de l'eau de vie.

7 L'animal sacrificiel (kurban) est soit offert par l'ancien roi, soit acheté avec l'argent collecté par les vignerons. Le sacrificateur qui égorge le veau ou le mouton est un vieux devin, riche en bétail ou en terres. Il est élu à vie par toute la communauté masculine du village et officie à chaque sacrifice collectif. L'animal sacrificiel cuisiné par les gardes champêtres doit être de préférence rôti à la broche, car le rôti dégage plus de fumée odorante que le bouilli et il est bien connu que Tryphon, comme d'ailleurs toutes les divinités, est friand de senteurs culinaires. Cependant, si l'animal sacrifié est trop grand $^{5}$ et donc ne peut être rôti entier ${ }^{6}$, on le découpe en gros morceaux et on le fait bouillir dans son chaudron.

8 Au cours du repas accompagné de chants et de danses, l'ancien roi déclare par trois fois son abdication. À la troisième fois, sa démission est admise et l'on choisit le nouveau tryphon. Une fois le repas terminé, on brûle les os et les restes ${ }^{7}$ et on enterre une bouteille de crème et parfois quelques morceaux de viande sous la pierre sacrificielle. Ensuite on part pour le village, les musiciens en tête, suivis par le char du roi tiré par des hommes (ou bien la charrette tirée par des chevaux). Le roi, entouré de ses gardes du corps, porte une couronne de sarments sur la tête ainsi que, comme tous les participants, un cerceau de sarments sur l'épaule. Au village, la procession s'arrête devant chaque maison et le propriétaire de la maison sort un chaudron de vin et offre à boire au cortège: d'abord au roi, ensuite aux autres hommes. Ce qui reste dans le chaudron est versé sur la tête du roi. Arrivé dans sa maison, trempé jusqu'aux os, le roi se change et un autre festin commence, cette fois-ci entièrement aux frais du nouveau tryphon. Les danses et les beuveries continuent jusqu'à la tombée de la nuit. Tout le monde, et le roi en premier, doit être ivre à la fête de Tryphon ${ }^{8}$.

Pendant que les hommes festoient dans les vignes (car les femmes n'y sont pas admises), des garçons de sept-huit ans, accompagnés de femmes, "tryphonisent » les arbres stériles. Pour ce faire, le jeune garçon brandira une hache devant un tel arbre en s'écriant : «Veux-tu donner des fruits, ou je te coupe !» Et la femme fera semblant de défendre "sa consœur végétale " par des gestes et paroles appropriés: «Ne le coupe pas, mon garçon, il produira cet été, j'en suis sûre !» Cette scène sera répétée trois fois devant chaque arbre. Le même effet pourra être obtenu si l'on frappe l'arbre avec des sarments. 

sévères que ceux des fêtes des loups d'automne (du 11 au 21 novembre) ${ }^{11}$, les Lupercalis bulgares - les automnales et les vernales ${ }^{12}$ - s'accordent parfaitement sur la prohibition impérative du tissage, du filage, du lavage du linge et des vêtements, de la couture et de l'évocation du nom du loup.

Lorsqu'il s'agit d'une femme stérile, soit une vieille matrone la soignera en brandissant le dévidoir", soit le mari à son retour le soir la «tryphonisera » à coups de poing ${ }^{10}$.

Il est possible aussi, le même jour, par le même procédé, de conjurer les sorcières qui retirent le lait des vaches et font avorter les femmes enceintes. Et c'est encore une vieille qui s'en chargera en allant à l'étable pour frapper avec une hache l'auge et les poutres. En faisant ceci, elle s'écrie : « On n'entend rien, parce qu'il y a trop de beurre et de fromage! »(Arnaoudov, 1972, 216 ; Xajtov, 1958).

\section{FERMER LA gUEULE DU LOUP}

Il est clair que la tryphonisation, à l'instar de l'élagage « en vrai », a pour but de faire fructifier les vignes, les arbres... et les femmes.

Toutefois, à la différence de la taille profane, la tryphonisation est un acte symbolique : à la fête de Tryphon Zarezan on ne coupe que neuf (ou douze) sarments de chaque vignoble, et on fait semblant de couper les arbres et les femmes.

Car le $1^{\text {er }}$ février, comme les deux autres «tryphons » (trifunci) suivants, on ne travaille ni dans les champs ni à la maison. Ces jours-ci sont d'ailleurs doublement fériés : d'une part en l'honneur de Sa Sainteté l'Ivrogne Camus, et d'autre part en l'honneur des loups.

Ainsi, il est fortement déconseillé pendant les trois tryphons d'ouvrir les ciseaux et le couteau pliant : il est même souhaitable, pour fermer la gueule des loups, de ficeler ces instruments avant de les ranger dans un endroit inaccessible. Certains poussent le zèle à tel point qu'ils ne se servent d'aucun instrument tranchant.

Toujours pour neutraliser les loups, une recette de bonne femme préconise de coudre ensemble le devant et le derrière des ourlets des jupons. Pour la même raison, il est bon de renverser le mortier et le pétrin.

Il est dangereux, croit-on, de filer, tisser ou coudre, surtout un vêtement masculin, pendant ces fêtes, car l'homme qui le portera sera dévoré par les loups.

Quant au tabou du nom de la bête, il est bien connu partout en Europe, que « lorsqu'on parle du loup, on en voit la queue $»^{13}$.

À quoi correspondent ces deux Lupercales?

Selon les croyances populaires (Marinov, 1914, 325) chaque louve s'accouple avec une douzaine de mâles pendant les fêtes des loups automnales qui marquent le début de l'hiver ${ }^{14}$. Durant cette période d'activité sexuelle débordante, les loups sont terriblement agressifs, et plus particulièrement le loup boiteux, androphage forcené, nommé Kuculan ${ }^{15}$. Or la fête de Kuculan le 21 novembre " coïncide » avec le jour de la Présentation de la Vierge, d'où l'attribution du patronage des Lupercalia automnale à Notre-Dame-des-Loups (Vǎlčata bogorodica).

La durée de la gestation des louves étant de soixante-quinze jours environ ${ }^{16}$, leur parturition aura lieu aux Lupercales vernales. Or, le deuxième Tryphon (2 février), est 
le jour de la Purification de la Vierge qui, de ce fait, s'avère protectrice non seulement des femmes, mais aussi des louves en couches!

L'étrange apparition de la Vierge chaque fois qu'il est question de loups nous amène à soupçonner que le parrainage de Tryphon aux fêtes des loups en février est quelque peu douteux. D'autant plus que le saint ne se manifeste nullement au cours des Lupercalia automnales.

24 Les ethnologues et les folkloristes bulgares n'auraient-ils pas raison de tenir le jumelage curieux de la fête viticole avec les Lupercalia pour une juxtaposition hasardeuse de deux complexes rituels indépendants? L. Peneva-Sabeva $(1971,63-64)$ va même plus loin lorsqu'elle affirme que la tryphonisation de la treille avec une hache, observée dans les villages de Kamena et Javornica (Bulgarie du Sud-Ouest), tryphonisation accompagnée d'une flagellation avec des sarments, serait une transposition de la coutume arboricole de Noël. Il est vrai qu'à Noël on menace les arbres fruitiers comme on le fait à la Saint-Tryphon. Mais les «sales jours » (mrăsni dni) entre Noël et l'Épiphanie sont encore une période pendant laquelle sévissent les loups et beaucoup d'autres esprits infernaux. Et puis la Vierge n'est-elle pas pour quelque chose dans la naissance de Jésus-Soleil ? N'est-elle pas aussi impliquée dans le mythe qui « explique » pourquoi Tryphon Zarezan est devenu camus?

\section{COMMENT SAINT TRYPHON S'EST COUPÉ LE NEZ}

Il n'y a pas en Bulgarie de protocoles qui assignent des lieux, un temps ou des manières précises pour la récitation des contes et des mythes. Les narrateurs dans la plupart des cas sont des femmes qui ont toujours les mains occupées lorsqu'elles racontent des histoires ${ }^{17}$. C'est pourquoi la récitation, en règle générale, n'est pas accompagnée de gestes narratifs. Le mythe de Tryphon-le-Coupé (Tryphon Zarezan), centré justement sur un geste, en est une exception. On raconte ce mythe invariablement à la SaintTryphon, lors des repas communs et des réjouissances. Mais il peut être communiqué à n'importe quel moment de l'année au cours d'une conversation qui touche de près ou de loin les vignes. Il serait raconté plutôt par des hommes, car la taille des vignes, commentée par le mythe, rituelle ou profane, est une affaire d'hommes.

Dans la littérature ethnographique bulgare qui n'attribue à aucun récit populaire le statut de mythe, l'histoire de Tryphon au nez coupé est classée dans la rubrique des légendes ou bien dans celle des contes hagiographiques. Quant aux usagers qui ne font aucune distinction entre mythe, légende ou conte (pour la bonne raison que la tradition orale ne les perçoit pas comme genre différencié), ce récit est une prikazka (conte, histoire). Mais il s'agit bel et bien d'un mythe, puisque le récit se propose (et il parvient à sa façon) d'expliquer l'origine d'un rite.

Je rapporte ici la version que j'ai entendue pour la dernière fois à Suxindol en 1968 :

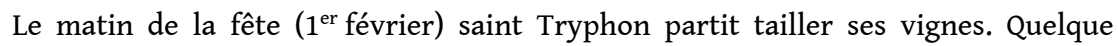
temps après, lorsqu'il était en train de travailler dans son vignoble, il vit s'approcher la Vierge avec son bébé le Christ.

- Tiens, la putain avec son bâtard ! Où est le père de cet enfant ? - s'écria-t-il.

Maria, profondément vexée, décida de se venger. Elle retourna précipitamment dans le village pour se rendre tout droit dans la maison de son offenseur.

- Va vite aux champs! - dit-elle à la femme de Tryphon. - Ton mari s'est coupé le nez en taillant les vignes.

L'épouse affolée accourut au vignoble où elle vit son mari sain et sauf. 
Tryphon étonné l'interroge sur l'objet de sa venue inattendue. La femme lui rapporte les paroles de la Vierge.

- Maria, cette menteuse, elle t'a raconté des bobards ! - s'indigna Tryphon. Je ne suis pas fou! Je sais comment tailler les vignes! Je fais «comme ça » et non pas « comme ça!"

Sur ces mots, il se coupa le nez. Quelques gouttes de sang tombèrent sur les racines du cep qu'il était en train de tailler. C'est de ces gouttes de sang que provient la couleur rouge du vin. Depuis, à la Saint-Tryphon, on coupe trois fois trois sarments de trois ceps dans la vigne, en versant du vin sur leurs racines.

Ce texte demeure, évidemment, incompréhensible, si l'on n'explique pas les gestes faits par Tryphon et reproduits par le narrateur.

En disant "comme ça ", le conteur qui tient dans sa main une serpe imaginaire la déplace horizontalement tandis que lorsqu'il prononce les mots « et non pas comme ça », il lève brusquement la serpe verticalement et... « coupe son nez ».

Ces deux gestes, l'un technologiquement correct, l'autre "faux ", s'inscrivent dans un protocole gestuel commun à tous les peuples balkaniques. Ainsi encore, l'affirmation («oui!») s'exprime dans les Balkans par un mouvement horizontal de la tête, et la négation («non ») par un mouvement vertical.

Comme le rite de la taille des vignes n'existait pas encore, et comme nous sommes en période lupercale, les gestes de Tryphon - le «faux » et le «vrai »- sont de toute façon des signes d'actes prohibés. C'est en tout cas ce qui ressort de la variante de notre mythe enregistrée au début du siècle par Deržavin en Bessarabie ${ }^{18}$. Selon cette variante, Tryphon le maraîcher s'est coupé le nez parce qu'il travaillait pendant les jours fériés. La Sainte Vierge l'avait prévenu, mais il passa outre.

Pour ces Bulgares expatriés probablement depuis quelques générations ${ }^{19}$, les Tryphons (trifunci) n'étaient que des fêtes des loups. Ce qui explique leur version appauvrie.

Dans toutes les autres variantes connues, recueillies pour la plupart vers la fin du XIXe siècle, le conflit «personnel» du génie des vignes avec la déesse vindicative est au premier plan. Assez curieusement d'ailleurs, aucune de ces variantes ne s'écarte de la trame du récit que nous avons proposé comme mythe de référence. En réalité ces variantes ne font qu'ajouter quelques précisions, mais ces précisions sont de taille!

la version provenant de la région de Tirnovo (Bulgarie du Nord-Ouest), rapportée par Slavejkov (1896), nous apprend que la Vierge elle-même s'est rendue fautive pour ne pas avoir respecté la quarantaine imposée par la coutume aux femmes après l'accouchement. En effet, en Bulgarie, comme ailleurs, la femme "souillée » par l'accouchement est tenue pour impure et vulnérable pendant une période de quarante jours ${ }^{20} \ldots$ d'où la Purification de la Vierge le quarantième jour après Noël, le 2 février, appelé dans toutes les langues slaves Stretenie, c'est-à-dire "l'heureuse rencontre " ${ }^{21}$.

Mais voyons plutôt comment se sont passées les choses d'après la variante mentionnée :

La Sainte Vierge au lieu de se faire purifier comme l'exige la coutume le 2 février,

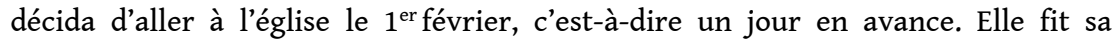
toilette, tressa ses nattes, prit l'Enfant Jésus et partit pour l'église.

Sur le chemin elle fut rattrapée par saint Tryphon, qui le même jour avait décidé d'aller tailler ses vignes. Lorsqu'il vit la Vierge, comme elle était quelque peu sa parente, il n'a pas pu se retenir de se moquer d'elle :

- «Tiens, Maria! La voici telle une chienne avec son chiot, elle traîne son bâtard! Où vas-tu ? Où est le père de cet enfant ? » Il dit ces mots et s'éloigna. 
La Sainte Vierge, affligée de ces paroles, des choses que les hommes pensaient et disaient d'elle et de son enfant, décida de ne plus aller à l'église. Sur le chemin de retour, en passant devant la maison de Tryphon, elle vit sa sœur, l'appela et lui dit : «Ma jolie, va porter à Tryphon du sel et des chiffons (pansements), car il s'est coupé avec la serpe... »

\section{pensées secrètes :}

Saint-Tryphon était vigneron et avait beaucoup de vignes. On dit qu'il s'est coupé lui-même le nez. Un jour il alla dans ses vignes et travailla jusqu'à midi. Après midi, sa sœur la Vierge est allée cueillir de l'oseille et en faisant ceci se mit à chanter. Saint Tryphon ne l'a pas reconnue et en pensant que c'est une autre femme, s'écria : « 0 ! 0 !» La Vierge l'entendit, reconnut son frère et pensa qu'il s'était coupé le nez. Elle retourna vite à la maison, prit des pansements et revint au vignoble. Son frère lui demanda pourquoi elle est venue. La Vierge lui répondit qu'elle avait pensé qu'il s'était coupé le nez. Alors Tryphon brandit sa serpe et se coupa le nez.

On dit que Dieu l'avait puni parce qu'en pensant qu'il s'agissait d'une autre femme, il eut envie de sa sœur.

(Tanović, 1939)

\section{D'autres textes, comme celui recensé par K
sans détour le désir scandaleux de Tryphon}

Un jour Tryphon partit avec sa sœur la Vierge pour tailler les vignes. Sur le chemin, il resta un peu en arrière et en regardant sa sœur dit: "Ma sœur, elles sont blanches tes jambes! » Elle lui répondit : «Oh, frère, que tu te coupes le nez!» Ils sont arrivés au vignoble. À l'instant même où saint Tryphon se mit à tailler les vignes, il se coupa le nez. Dieu l'avait puni pour ce qu'il avait dit à sa sœur. 


\section{MA SœUR, MA BIEN-AIMÉE...} nullement la Vierge : plusieurs versions, nous l'avons vu, sinon toutes, insistent sur le fait que la déesse avait prémédité ce châtiment. Mais de toute façon, quelle que soit son attitude personnelle, la Vierge agit dans ce cas en parfait accord avec la morale sociale. Et la société a d'excellentes raisons pour ne pas tolérer l'amour entre personnes de même sang, qui corrompt la politique des échanges matrimoniaux. Quelquefois, elle met dans la même enceinte les amours d'individus de même sexe. La preuve: les homosexuels bulgares ont comme " patronne »... la Sainte Vierge ! Ainsi le 2 février au matin, les adeptes des amours particulières de Sofia devaient se rendre avec une couronne de pain au sésame ${ }^{22}$ et un tube de vaseline aux bains publics de Gorna- 
Banja $^{23}$... pour se «purifier » j'imagine... Et on entend souvent la veille de la fête, des hommes se taquiner mutuellement: "On se voit demain à Gorna Banja? " ${ }^{24}$ Comme quoi les Jörai ne seraient pas les seuls à penser que « coucher avec ma sœur » et « aimer une personne de mon sexe ", c'est du pareil au même ${ }^{25}$.

Peut-on imaginer un monde morcelé en familles endogames, ou bien en communautés homosexuelles, donc stériles? Ou encore une humanité qui s'aime n'importe comment... à la manière des bêtes sauvages?

51 Notre mythe et les pratiques rituelles tryphonesques explorent à la fois tour à tour et simultanément ces dangers imminents.

Il s'agit bien sûr, également, d'une taxinomie des êtres vivants établie suivant des critères de reproduction, à savoir :

- reproduction hétérosexuelle exogame ;

- reproduction parthénogénétique ou « homosexuelle » à la manière des plantes (la vigne).

Cette division antinomique est doublée d'une seconde dichotomie d'ordre alimentaire :

- consommation de nourriture fraîche, donc non élaborée, d'origine soit végétale, soit animale ;

- consommation de «charogne » végétale et animale, donc élaborée, à la manière des plantes nécrophages.

Situés au carrefour de ces deux chemins impossibles, les héros tryphonesques (Dionysos, Tryphon, Atys, les Titans, Lycurgue, etc.) choisissent l'un ou l'autre, en fonction de leurs goûts et pratiques « alimentaires » : (voir figure page suivante).

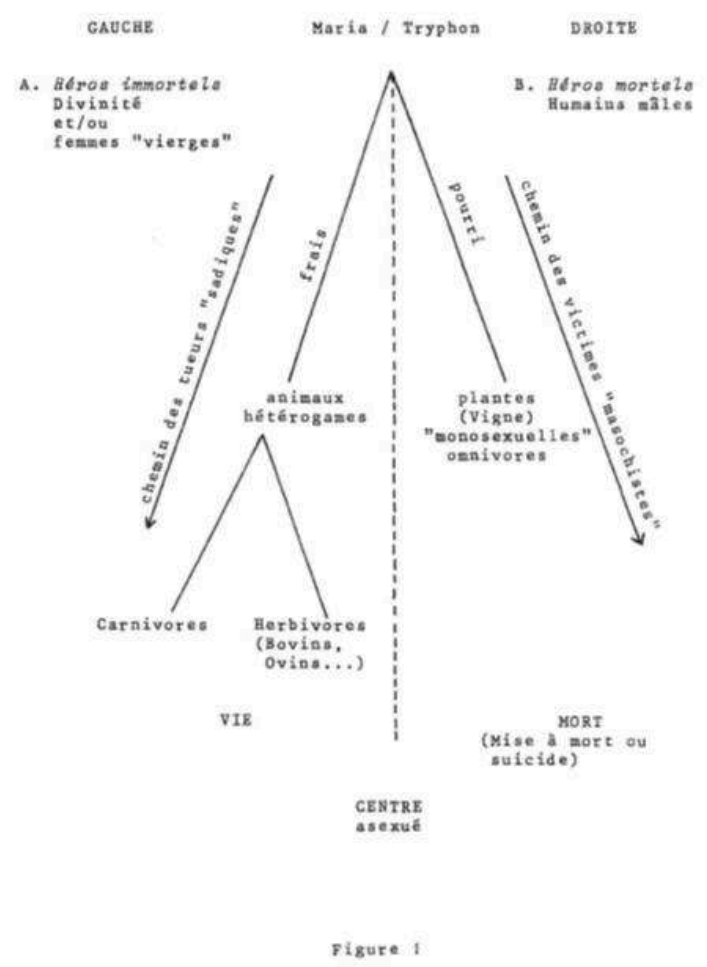




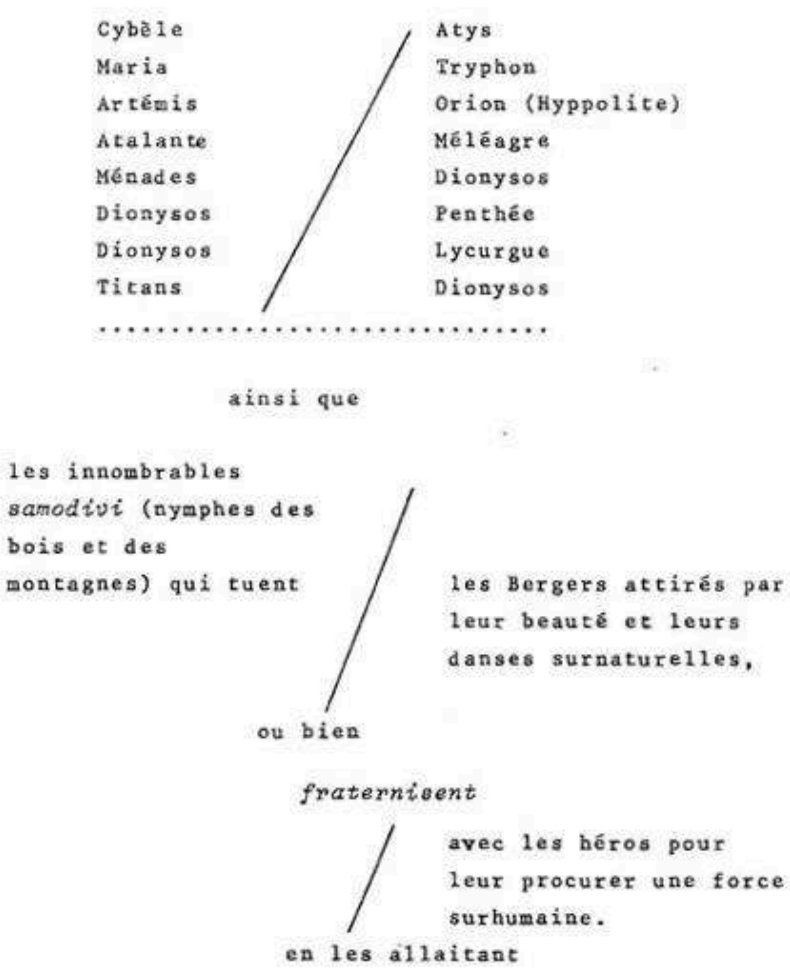

Et le pacte de lait que les samodivi concluent avec de jeunes gens, fait pendant au pacte de sang que les humains (femmes avec hommes, ou hommes avec hommes) concluent dans la vie courante. Une telle sœur adoptive (posestrima) ${ }^{26}$ est tenue de porter secours chaque fois que son frère adoptif (pobratim) ${ }^{27}$ est en danger.

Comme n'importe quelle sœur.

\section{QUI A PEUR DE VIRgINIA WOOLF...}

Le pouvoir d'engendrer, donc de dispenser la vie, n'est pas simplement une propriété que les femmes partagent avec les dieux. Mieux encore: c'est une fonction intrinsèquement féminine que les dieux mâles s'approprient chaque fois qu'ils sont poussés par le désir d'enfanter. Et la valorisation sociale du fameux instinct maternel n'est-elle pas dans une certaine mesure la projection du désir masculin d'être mère? Car la femme l'est toujours - réellement ou virtuellement comme ces vierges divines, maitresses des animaux sauvages qui président en même temps à l'accouchement (Artémis, Maria). Ou bien comme les samodivi, nymphomanes et vierges ${ }^{28}$ qui ont cependant souvent des enfants ${ }^{29}$.

Aucun mâle, même Zeus ne peut procréer sans femme. Ainsi, pour accoucher d'Athéna qu'il fait sortir de sa tête, il a fallu quand même qu'il avale Métis, enceinte d'elle. Ou bien, pour mettre au monde Dionysos, que Zeus porta cousu dans sa cuisse, il a fallu l'extraire du corps foudroyé de Sémélée ${ }^{30}$. Et il utilise des procédures similaires lorsqu'il foudroie les Titans pour avoir dévoré Dionysos. Le cœur de l'enfant divin, laissé de côté par les cannibales chtoniens, fut dérobé par Athéna. Cette sœur «extra-utérine "si j'ose dire de Dionysos, déroba le cœur pour le donner à Zeus. Après l'avoir avalé, l'Olympien fut à même d'engendrer Dionysos avec Sémélé-Zemêlo (encore une 
chtonienne !) ${ }^{31}$ qu'il foudroya pour enlever le fruit de son giron et le replanter dans son propre corps.

Dans le premier épisode de ce mythe viticole les Titans apparaissent comme instrument de la génération artificielle (masculine) manipulée par Zeus, génération pour laquelle il n'a pas pu se passer du corps d'un être féminin ou efféminé. En effet, dans cet épisode les génies chtoniens se conduisent comme des femmes lorsqu'ils font bouillir, ensuite rôtir les chairs de leur victime. Car contrairement à ce qu'affirme M. Detienne :

... la cuisine à laquelle se livrent les Titans est étrangement fantaisiste : ils rôtissent les chairs de la victime qu'ils ont préalablement bouillies; Dionysos est transformé en rôti bouilli. Pris en lui-même, le mythe orphique est un discours paradoxal...

(Detienne, 1977 : 29)

à la suite de C. Lévi-Strauss :

Dans les temps anciens, disaient les Grecs, par la bouche d'Aristote, les hommes rôtissaient tout ». Il s'ensuit qu'on peut faire bouillir une viande préalablement rôtie, mais non faire rôtir une viande déjà bouillie, car ce serait aller contre le sens de l'histoire...

(Lévi-Strauss, 1968 : 398)

Cette procédure «fantaisiste» dans les Balkans est une technique culinaire féminine tout à fait banale. Le poulet au riz que les épouses préparent la veille de la fête de SaintTryphon pour le festin champêtre des vignerons n'est pas fait autrement. Il ne va pas de même pour la seconde partie du menu tryphonesque, composé d'un mouton rôti ou veau bouilli sur le lieu de sacrifice (obročište) ${ }^{32}$, sans adjonction de céréales ni de légumes. En règle générale, chaque fois que l'on fait un sacrifice collectif, l'animal sacrificiel est soit rôti soit bouilli ${ }^{33}$, sans adjonction d'ingrédients d'origine végétale et ceci toujours à l'extérieur de la maison ${ }^{34}$. Il s'ensuit que les différences principales entre la cuisine féminine et la cuisine masculine se réfèrent d'une part au lieu de la préparation (dans la maison ou à l'extérieur), et d'autre part à l'origine végétale et/ou animale des ingrédients. Ainsi les plats sacrificiels, exclusivement carnés, des hommes sont confectionnés collectivement et toujours sur un lieu public, tandis que la cuisine profane féminine, végétarienne ou mixte, est toujours domestique. Le problème du mixage concerne également les techniques culinaires masculines ou féminines : ainsi les hommes ne font que rôtir, ou bouillir, ou bien tout simplement griller leurs viandes, tandis que les femmes, pour la confection d'un seul plat utilisent toutes sortes de techniques raffinées et variées ${ }^{35}$. Sur le plan culinaire, les femmes se conduisent un peu comme les plantes nécrophages qui consomment des aliments élaborés d'origine végétale et animale, tandis que la cuisine sommaire sélective et sauvage des hommes se rapproche du mode de consommation des animaux «omophages ». Autrement dit, les hommes comme les fauves tuent leur nourriture fraîche, ou bien à l'instar des dieux (Zeus notamment) la brûlent, alors que les femmes et les plantes transforment les aliments pourris en matière vivante. Faut-il rappeler que les femmes depuis toujours et partout ont une prédilection pour la cuisson par ébullition - procédure qui implique l'adjonction de liquides et qui, sur le plan symbolique, est associée à la putréfaction et à la résurrection? Dans cet ordre d'idées n'oublions pas non plus que la cuisine féminine nécessite toujours un récipient - ce qui «explique» en l'occurrence pourquoi les femmes balkaniques n'utilisent qu'incidemment la technique éminemment masculine du grillage ${ }^{36}$.

Par le truchement de ce récipient - pot, marmite, chaudron-enclavé dans le foyer domestique, comme l'est l'utérus dans le corps féminin, la théorie botanique et culinaire réussit à englober le processus de la gestation. Les multiples précautions 
prises par la femme enceinte, lorsque la graine semée dans son corps se transforme en fruit ${ }^{37}$ illustrent bien cette assimilation. Ainsi, à partir du quatrième mois, la future mère commence à porter autour de ses reins une ceinture de sarment de vigne blanche $(\text { clématite })^{38}$; et elle glisse dans son corsage une pomme ou une poire, qu'elle gardera jusqu'à la délivrance. Ce fruit ne doit pas être cueilli, mais ramassé au pied de l'arbre. Ensuite, une fois que l'enfant est mis au monde, le premier souci de la sage-femme sera de le faire passer par l'ouverture du trépied (pirustija) - symbole du sexe féminin ${ }^{39}$ ainsi que de le faire tenir quelques instants au-dessus du feu (Arnaoudov, 1943: 603), autrement dit de faire « griller » l'enfant déjà « bouilli ».

61 Cette théorie végétative, prise en charge par les pratiques rituelles viticoles, culinaires et d'accouchement, a trouvé quelque vingt-cinq siècles auparavant sa formulation verbale dans les écrits d'Hippocrate :

... La nutrition et la croissance de l'enfant a lieu lorsque se trouve dans la matrice ce qui provient de la mère (...). De même, les plantes se nourrissent de la terre et telle est la terre, telles sont aussi les plantes. Quand elle est semée, la graine se remplit de l'humeur qui provient de la terre (...). Quand la plante, avec le temps, est plus solide et mieux enracinée, elle a des veines larges vers le haut et vers le bas, et alors ce qu'elle tire de la terre n'est plus aqueux, mais plus épais, plus gras et plus abondant. Cette humeur, échauffée par le soleil, se met à bouillir ${ }^{40}$ aux extrémités et devient fruit, selon l'espèce à laquelle la plante appartient (...). Après l'ébullition, le fruit est nourri par la plante; car la plante tire de la terre et transmet au fruit. Le soleil cuit et affermit le fruit, en attirant à lui sa partie la plus aqueuse (...)

(1970, XXII)

Et quelques paragraphes plus loin, l'éminent guérisseur ${ }^{41}$ de l'Antiquité conclut : « Celui qui veut réfléchir à ce qui a été dit à ce sujet trouvera que, du début à la fin, la croissance naturelle des plantes et de l'homme est tout à fait semblable.» (1970, XXVII).

La fabrication du vin à partir du raisin foulé, puis mis à fermenter, c'est-à-dire à bouillir $^{42}$ dans des cuves déposées dans les caves des maisons s'inscrit, d'une façon évidente, dans la symbolique végétale de l'enfantement conjuguée à la technologie culinaire. Ainsi, pour les vignerons bulgares, la durée de la fermentation, qui commence vers l'ouverture de la saison des mariages du 14 octobre, est de quarante jours. Or, le même délai de quarante jours est nécessaire, selon Hippocrate (1970, XVIII) pour la transformation de la graine humaine en fœtus. La formation du vin donc se termine juste après les Lupercalia automnales. C'est à cette époque - théoriquement vers le 25 novembre - que l'on procède à la première « purification » (soutirage) du vin, et la seconde, « comme par hasard » aura lieu deux quarantaines de jours plus tard au moment de la Purification de la Vierge, le 2 février! Deux autres purifications auront lieu en mai et en août, et ce n'est qu'à partir du 14 octobre que l'on aura le droit d'entamer le vin nouveau (Peneva-Sabeva, 1971). Le rythme de quarantaines, ou plus exactement de six hebdomades (42 jours) est un rythme calendaire d'origine «biologique».

64 La fermentation et la maturation du vin comme le signale W. F. Otto $(1969,156)$ sont associées également au cycle évolutif de la vie humaine :

Le mystérieux processus de la fermentation et de la maturation du vin a aujourd'hui encore le pouvoir d'évoquer chez les vignerons et les connaisseurs des représentations qui rappellent de loin la pensée mythique. Ils considèrent le vin comme un être vivant qui se forme progressivement en évoluant du bouillonnement chaotique de la jeunesse à la limpidité et à la force de la maturité. 
Dans cet ordre d'idées les soutirages successifs suivis par la consommation (la « mort ») du vin, c'est-à-dire l'eucharistie, correspondraient aux différentes étapes de l'évolution de la vie humaine marquées par les rites de passage ${ }^{43}$.

Or, la préparation du vin, et en général des boissons spiritueuses et spirituelles ${ }^{44}$ relève d'une technique masculine qui, à la base de substances végétales, reproduit cette humeur subtile, composante éphémère et ignée de la sève, du sang et du sperme qui est le souffle vital ou l'esprit.

Et si pour obtenir du vin on doit faire bouillir le jus des fruits, la préparation de l'alcool (la quintessence !) exige une opération supplémentaire qui est le rôtissage. En effet, les Bulgares disent, c'est l'expression courante, qu'ils font rôtir leurs alcools (peka rakija)... Autrement dit, la cuisine bizarre des Titans n'est qu'une distillation de « l'esprit » pur...

68 À cette étape de l'analyse, les interprétations proposées ici rejoignent la théorie de Cl. Gaignebet sur la " circulation des souffles » (1974, chap. VII) et le carnaval européen, carnaval qui, selon l'auteur, « remet symboliquement en question le grand privilège de la femme : engendrer l'enfant » (1974, chap. I, 16).

69 Avant d'aborder la problématique carnavalesque centrée sur la date fatidique du 2 février, je voudrais rapporter ici une dernière version du mythe de Tryphon au nez coupé, version qui apparaît comme une réplique à peine déformée du conflit de Dionysos avec Lycurgue. On se souvient que, dans le récit homérique, le roi thrace s'opposait farouchement au culte dionysiaque. À la suite d'une série d'affrontements, Dionysos parvint à lui troubler l'esprit à tel point qu'il prit son propre fils pour un pied de vigne. Ainsi, en pensant qu'il taillait ce cep, Lycurgue abattit son fils avec une hache. Mais voyons plutôt comment se passent les choses dans le mythe bulgare :

Le premier jour du mois du Coupeur (février) ${ }^{45}$, saint Tryphon prit dans son sac un pain et une gourde de vin, accrocha la serpe à sa ceinture, et se mit en route pour aller tailler ses vignes. Chemin faisant il rencontra la Vierge qui le salua :

- Bonne santé, saint Tryphon, Si Dieu le veut, où vas-tu?

- Je vais tailler les vignes, Sainte Mère de Dieu, dit-il.

- Il vaudrait mieux de ne pas y aller ni aujourd'hui, ni demain, ni après-demain. Je me prépare à aller demain à l'église faire dire ma prière. Et je ne veux pas que les hommes te célèbrent le troisième jour du mois du Coupeur. Je veux que tu sois avant moi, et non pas après.

- Pas question, Mère de Dieu, j'ai pris mon pain, ma gourde de vin et j'ai aiguisé ma serpe.

Ayant compris que Tryphon ne voulait pas l'écouter, la Vierge lui dit:

- Bon, vas-y, Tryphon. Mais sais-tu tailler les vignes?

- Et comment, bien sûr que je sais !

- Bon, s'il en est ainsi, vas-y, fais voir comment tu tailles les vignes !

- Eh bien, Mère de Dieu, voilà !

Tryphon sortit sa serpe, et, ayant pris son nez pour un sarment, le saisit, le coupa et resta camus.

C'est alors que le pauvre Tryphon comprit qu'il s'était coupé le nez parce qu'il n'avait pas écouté la Vierge. Ainsi, il devint camus, et sa fête fut mise avant celle de la Vierge, et non après...

(Cepenkov, 1900, 291-292)

La parenté sémantique entre la taille, la castration ou le châtiment subis par le héros contestataire ou bien par la vigne, dans les deux récits, ne laisse aucun doute : tous ces vocables dérivent du verbe latin castrare qui signifie mutiler (châtier) et... purifier. L'inversion des rôles de Dionysos-Tryphon, bourreau dans le mythe grec et victime dans le texte bulgare est parfaitement normale pour un dieu-bâtard fils d'une humaine 
et de surcroît virtuellement bi-sexuel. Ainsi face à un humain, son côté féminin et divin prend le dessus, tandis que chaque fois qu'il affronte une déesse, il redevient homme mortel.

Le nom de l'illustre adversaire de Dionysos, Lycurgue, évoque en filigrane la présence du loup. Ce nom lycamorphe rappelle un autre « loup-garou » de la mythologie grecque - Lycaon. La double transformation du roi anthropophage changé en loup, puis en constellation pour avoir défié l'olympien, s'inscrit parfaitement dans la geste dionysiaque : la constellation du Loup (Lycaon) qui forme une triade stellaire avec le Bouvier et la Vierge, est appelée également la Bête, la Lance du Centaure, ou la Panthère. Or, la Panthère, cette féline parfumée ${ }^{46}$, fille adultérine de la lionne et du $\operatorname{pard}^{47}$, ou bien tout simplement la femelle de cet animal nauséabond ${ }^{48}$, réputée, comme la louve, pour sa lubricité, est l'avatar et la comparse attitrée de Dionysos ${ }^{49}$.

Selon le témoignage de Pline (VIII, 23, 62) cette " allumeuse » (car la panthère séduit pour tuer), "avait sur le poitrail une tache semblable à la lune, qui s'arrondit et se creuse en croissant avec elle ». Lorsqu'on se souvient que les maitresses des animaux sauvages (Artémis, Maria...) sont les mêmes vierges qui président au processus utérin, donc nocturne, de la germination-gestation des animaux, des humains et des plantes, cette association avec la lune n'a rien d'étonnant.

Et le seul moyen de se soustraire aux charmes périlleux de la Panthère-Louve, et par là aux sortilèges des Vierges redoutables, c'est de retourner contre elles, comme le note M. Detienne, « leur pouvoir de séduction et de tromperie » en se servant du vin. Pour ce faire, poursuit l'auteur en livrant les recettes «éprouvées » des anciens (Oppien, IV, 320-353), «il suffit d'en répandre quelques flacons à proximité d'un point d'eau. Alertées par l'odeur, les panthères s'approchent et boivent autant qu'il y en a. Il ne reste plus alors qu'à s'en emparer en profitant de leur ivresse » (Detienne, 1977, 96).

\section{POUR MÉNAgER LE BœUf ET LE LOUP}

On n'a nullement besoin de se référer aux calendes grecques pour dénicher les loups de la tradition populaire bulgare: toute la période hivernale à partir des Lupercalia automnales qui marquent le commencement du carême de l'Avent jusqu'au début du jeûne pascal, est dominée par les fauves. Ainsi, la durée de la période carnivore qui succède au carême de l'Avent est en corrélation « directe » avec la fécondité des louves. En effet, le nombre des louveteaux qu'une louve met bas lors des tryphons est égal, diton, au nombre des jours carnés depuis Noël jusqu'à la Quinquagésime. La semaine qui suit la Quinquagésime, appelée «semaine de fromage» dans les pays orthodoxes, est vouée au végétarisme modéré: la nourriture carnée est prohibée, mais la consommation d'aliments d'origine animale - œufs et laitages - est au contraire vivement recommandée. Le poisson, les escargots, les moules et autres animaux à sang froid comestibles, classés dans une catégorie intermédiaire entre les espèces animales et les végétaux, font également partie du régime alimentaire de la semaine du fromage. Ensuite, pendant le carême leur consommation sera évidemment interdite.

Pendant toute la semaine jusqu'au samedi du carême-prenant (sirni zagovezni) ${ }^{50}$ les jeunes gens et les jeunes filles se balancent, flirtent, dansent. Après cette date, tous les jeux sexuels seront suspendus pour une quarantaine de jours. Bien entendu la saison des mariages, comme partout en Europe, prend fin avec l'avènement du carême. Le 
jeûne absolu - alimentaire, sexuel et verbal - sera inauguré avec le festin et les feux du carême-prenant du samedi. Le repas festif - œufs, poisson, pâtes feuilletées, fromage, sucreries - sera célébré en famille. Il se termine, pour la grande joie des enfants avec la farce de l'œuf, du fromage ou bien du « halva blanc » (c'est-à-dire du nougat) suspendus à un fil de chanvre. La ficelle est fixée soit à une poutre du plafond, soit au bout de la quenouille ${ }^{51}$. Il s'agit d'attraper avec la bouche, les bras croisés derrière le dos, l'appât ébranlé. Ensuite, à plusieurs reprises on allume la ficelle pour présager l'avenir de la récolte, le bonheur de chacun des membres de la famille, la santé et la fécondité du bétail. Il est de bon augure que la ficelle s'enflamme et brûle vite. Après ce dîner, la nuit, les jeunes gens - garçons et filles - allument des feux sur les collines environnantes. Ils dansent et chantent des chansons, souvent très obscènes. Ces feux sabbatiques annoncent l'arrivée du carême et du carnaval qui aura lieu deux jours plus tard, le « lundi des chiens $»^{52}$.

Le terme qui désigne l'action de jeûner signifie littéralement « boviner $~_{53}$. Or, le même vocable signifie également «se taire en signe de respect $»^{54}$. Aussi, les kukeri (protagonistes du carnaval) sont-ils tenus d'observer le silence au cours de leurs performances. Ainsi le carnaval bulgare apparaît-il comme un mimodrame scandé par le tintement des sonnailles que les kukeri portent accrochées à leurs ceintures.

Sans rentrer dans les détails extrêmement riches et variés, signalons brièvement quelques traits pertinents de cette manifestation carnavalesque.

Le rite des kukeri, appelés aussi starci (vieux) est répandu principalement en Bulgarie orientale et centrale ${ }^{55}$. Comme ailleurs en Europe, les rôles principaux du carnaval bulgare sont joués par des hommes célibataires travestis. Leurs visages sont soit barbouillés de suie (sauf celui du roi), soit, et c'est beaucoup plus fréquent, portent d'énormes masques zoomorphes. Ces masques fantastiques surmontés de cornes ${ }^{56}$ figurent les deux croissants de la vieille et de la nouvelle lune, sont grotesques, mais pas du tout risibles : on dirait plutôt des monstres sortis des tableaux de Bosch. Ils sont fabriqués en peaux d'animaux herbivores (boucs, moutons, cerfs, lapins) ou bien de morceaux de calebasse, de toile, combinés avec d'autres matériaux résiduels. Certaines troupes comprennent des animaux réels: cheval, chameau ou bien ours ${ }^{57}$. L'habillement fantaisiste des kukeri se compose de costumes de femmes (pour ceux qui jouent des rôles féminins), de peaux d'animaux, de vieux sacs et de chemises. Il est à remarquer que même les kukeri "mâles " portent souvent des éléments du costume féminin - jupons et tabliers. Ils tiennent dans les mains des bâtons crochus, des gourdins, des épées en bois ou des ensouples avec lesquels ils frappent les spectateurs et les hôtes des maisons qu'ils visitent. En principe, les kukeri épargnent les femmes mariées $^{58}$, mais par contre, ils s'acharnent sur les jeunes filles.

Les personnages principaux de la troupe des kukeri sont le kuker-chef, appelé kuk/kuker tout court, ou bien "hadj", son "épouse " la Vieille (Baba), son auxiliaire l'Archer (pljufkač, strelnik), et, pour le troisième acte du spectacle, le roi.

80 Le kuker-chef qui porte le costume le plus pittoresque de la troupe est affublé d'un phallus en bois peint en rouge ${ }^{59}$. Il tient toujours en main un écouvillon avec lequel il barbouille le visage des spectateurs. Son « épouse ", la kukerica ou baba, est une « vieille bossue », souvent «boiteuse ». Elle porte en guise de bébé une bûche emmaillotée, ou bien elle est «enceinte ». La Vieille tient parfois une quenouille et file. L'Archer porte un arc et un petit sac plein de cendres accroché à la pointe de sa "flèche ». Avec cette "arme », il lance des cendres dans les figures des spectateurs. Pour le rôle du roi on 
choisit un homme marié avec des enfants vivants. Il ne porte ni masque, ni costume spécial. Son visage est blanchi et il a une barbe et des moustaches en laine blanche. De nombreuses « décorations » en fer-blanc sont accrochées à sa poitrine.

81 La troupe des kukeri comporte également un couple de jeunes mariés, des couples de fiancés, des collecteurs d'impôts, des tziganes-montreurs d'ours avec l'ours, des policiers, un pope, un médecin, des nègres (arapi) et autres personnages plus ou moins variables. Cependant, quelle que soit la composition de la troupe, la fonction, sinon le rôle de la mariée sont toujours présents. Ainsi, cette fonction est souvent assumée par la vieille.

Le carnaval s'ouvre tôt le matin par une poursuite des chiens ${ }^{60}$. Les kukeri (ou d'autres hommes) improvisent des lassos avec des fourches et des cordes, attrapent les chiens, les soulèvent, les balancent. Ils font suer les chiens de cette façon jusqu'à ce que les pauvres bêtes défèquent... du « halva »... Ce « halva » est ramassé dans des corbeilles et porté à travers le village "pour faire rire». Les excréments des chiens, animaux domestiques carnivores "purifiés » par pendaison le "Lundi pur» sont sans doute associés au halva suspendu du repas sabbatique du carême-prenant. Ce supplice purgatif est considéré comme une mesure prophylactique contre la rage ${ }^{61}$.

Ensuite les kukeri défilent dans les rues du village en dansant, en frappant et barbouillant les spectateurs. Ils arrêtent les passants et les obligent à payer une rançon. De temps à autre, le kuker empoigne par-derrière un spectateur pour simuler le coït. Il agit de même avec son "épouse». Si la Vieille est "enceinte», elle simulera un accouchement au cours du défilé matinal. Pour ce faire, elle montera sur un arbre et fera tomber d'en haut un chaton. Parfois cette délivrance a lieu en fin d'après-midi sur la place du village. Dans ce cas la kukerica s'emparera d'un spectateur pour le faire sortir de ses jupons en mimant les « douleurs » de l'accouchement. Si en cours de route les kukeri rencontrent une troupe étrangère (d'un autre village), des bagarres, parfois sanglantes s'ensuivent. Jadis, lors de ces rixes, des meurtres survenaient souvent. On enterrait les morts en dehors du village sans aucune cérémonie religieuse. La famille d'un kuker tué dans une telle bataille ne réclamait jamais vengeance.

Parfois en Thrace le défilé matinal est précédé par le mariage du kuker avec la kukerica ou bien par les noces collectives des fiancés. Le sacrement a lieu sur un tas de fumier (Arnaoudov, 1972).

L'après-midi du kukovden (jour des kukeri) est destiné aux visites. Au cours de ces visites des maisons, en commençant par celle du forgeron, les kukeri font la quête en dansant et en insultant leurs hôtes par des gestes et des coups. Les hommes leur donnent de l'argent et les femmes des victuailles et de la laine. Avec l'argent collecté et celui obtenu par la vente des produits, les kukeri achètent du vin pour leur festin. Lors de ces visites, les hôtes cherchent à enlever la Vieille pour la cacher. S'ils y parviennent, la bonne récolte, la fertilité et l'abondance seront assurées à leur maison. De ce fait cependant, le bonheur des autres maisons sera gravement menacé, car les kukeri, privés de la Vieille, ne pourront plus jouer. Pendant toutes ces embuscades la kukerica qui ne participe ni aux danses, ni aux bagarres ni à la quête, reste assise et file tranquillement sa filasse de chanvre.

Le dernier acte du spectacle se déroule sur la place du village vers la fin de l'après-midi. Il représente une cérémonie de labourage et de semailles présidée par le roi. Le souverain, qui n'intervient pas dans les manifestations diurnes, sera amené sur la «scène » dans un char tiré par la troupe. Le labour sera effectué soit par le roi lui- 
même, soit par le kuker ${ }^{62}$ avec une charrue tirée par les autres kukeri. Après le labourage, le roi sème quelques poignées de grains. Pendant le travail, le laboureur sera à plusieurs reprises culbuté, traîné et enfin « tué ». Le carnaval se termine par un festin des kukeri, évidemment végétarien. Les beuveries peuvent continuer quelques jours ${ }^{63}$.

\section{LA BOUFFONIE APHONE}

Aucune acrobatie philologique n'a réussi jusqu'à présent à expliquer d'une manière probante les termes kukeri, kukuovden (jours des kukeri). Pas de commentaire non plus du côté de la tradition orale. Aucun discours - ni mythe, ni conte, ni chant ne sont associés à ce carnaval muet. La seule et unique expression connue proclame que la fête des kukeri... n'existe pas (? !). En effet, la formule ironique na kukovden (à la fête des kukeri) signifie... jamais...

Cette fête énigmatique n'a pas manqué de susciter de nombreux commentaires. On n'a pas tardé, bien sûr, à évoquer les éléments d'une critique sociale dans la représentation caricaturale des noceurs-quêteurs: le pope, les militaires, le juge, les collecteurs d'impôts. Mais les personnages principaux - le kuker, la Vieille et le roi, ainsi que les éléments rituels échappent de loin à la glose sociopolitique.

On a cru pouvoir expliquer les mystères loufoques des kukeri comme un rite de fécondité et une séance de magie agraire où le symbolisme sexuel du labourage avec sacrifice mimé d'un esprit de blé serait préfiguré par la hiérogamie de la Vieille avec le kuker. La première interprétation dans ce sens fut proposée par l'éminent folkloriste bulgare Mixaïl Arnaoudov, auquel on doit l'inventaire le plus détaillé, ainsi qu'une exégèse très poussée de la fête (Arnaoudov, 1972, « Kukeri i rusalii »).

L'image caricaturale de cette «hiérogamie » ainsi que le sacrifice ludique sont dus, selon Arnaoudov à l'inévitable dégradation du rite jadis sacré :

Si le drame dionysien de sacrifice à un dieu se transforme en un simple carnaval et cela se produit lorsque l'ancienne religion disparaît - la cérémonie religieuse, dépourvue de signification, se dégrade en un jeu comique où le contraste immotivé de la joie et de l'affliction s'enrichit ${ }^{64}$ progressivement d'une multitude de détails.

(Arnaoudov, $1972: 93$ )

0 Toutefois, M. Arnaoudov a eu le mérite d'établir une correspondance entre trois fêtes importantes: la Saint-Tryphon, les mystères des kukeri et le rite des rusalii de la semaine qui suit la Pentecôte ou la Trinité. Dans sa quête d'ascendances prestigieuses, l'auteur fait remonter le rite de possession des rusalii vers les cultes agraires de CybèleDéméter-Dionysos et les mystères "chamaniques » des corybantes en passant par les Rosalia romaines. Pour Arnaoudov, la représentation de la mort et de la renaissance de l'esprit de la végétation ébauchée dans le labourage rituel des kukeri, trouve son apothéose dans la chute cataleptique et la « résurrection » des rusalii au cours de leurs danses extatiques. Quant à la fête de Tryphon-le-Coupé, certes d'inspiration dionysienne, elle serait la seule, dans sa spécialisation viticole, à porter l'empreinte d'une créativité autochtone ${ }^{65}$.

Le rite de possession bulgare n'a rien à voir avec la fête des fleurs laïque des Romains. Rusalia, comme le signale Arnaoudov lui-même (1972,13-137), est un terme archaïque ${ }^{66}$ qui désigne une fête populaire aux aspects carnavalesques. Et il n'y a point de roses dans la fête bulgare. Les crosses des rusalii sont surmontées de bouquets de dictame $(\text { rosen })^{67}$ et d'autres plantes réputées pour leurs vertus médicinales contre la folie, 
l'épilepsie, la paralysie et la fièvre. Au cours de leurs danses les rusalii muets agitent leurs thyrses pour exorciser les maux sacrés infligés par ces satanées Vierges, les fées des bois et des montagnes (samodivi).

Et pourtant Arnaoudov a raison: il y a une liaison intime entre les trois fêtes. Pour retrouver cette parenté, d'ordre végétarien, il nous faudra retourner au compte des louveteaux.

Dans le meilleur des cas la portée de la louve prolifique, qui met bas à la Saint-Tryphon, est au nombre de quarante/quarante-deux louveteaux.

Dans une telle année au printemps précoce, le carême-prenant modéré du Quinquagésime coïncidera avec les tryphons. La semaine des laitages débutera donc le 3 février. Une semaine plus tard, le 10 février, aura lieu la fête de la Peste (Čuminden), elle coïncidera avec le premier jour de carême absolu. Le carnaval des kukeri aura lieu de lendemain, à la Saint-Vlas (11 février). Or, « saint Vlas » est le nom «chrétien» de la Grande Divinité Bovine de l'antiquité slave: Volos/Veles ${ }^{68}$. Ce jour-ci, surnommé Mukovden, "jour de meuglement », on offre aux bêtes - bovins ou ovins - des pains rituels et du sel en meuglant. Il est interdit, à la Saint-Vlas, d'atteler les bœufs. Et d'ailleurs dans toute autre circonstance les « anges » (angeli), les boeufs jouissent d'un privilège spécial : on ne les tue jamais. Même les loups, dit-on, n'attaquent jamais un bœuf laboureur. Puisqu'il s'agit des deux aspects d'une même bouffonnerie, faut-il s'étonner que l'expression « au jour de meuglement» (na muk) signifie la même chose que la formule "à la fête des kukeri » : jamais...

95 La semaine des laitages se trouve donc bordée d'un côté par les loups (ou les louves en couche...) et de l'autre par des bêtes herbivores. C'est une semaine de confusion dominée par les femmes (la Vierge, la Peste...). Et il est bien connu que les femmes mélangent tout - pas seulement dans leur cuisine. C'est en raison de leur " goût " pour les mixages et en vertu de leur incapacité « congénitale » (et génitale) de différencier et d'ordonner que la pensée mythique bulgare les classe à la fois du côté des plantes et des animaux. Elles « sont » des louves et des vignes, des ourses et des vaches, carnivores et herbivores en même temps, tandis que les hommes sont soit l'un, soit l'autre, jamais les deux à la fois. L'élan naturel vers la confusion, exprimée par le flux de lait de la semaine des laitages qui nourrit indifféremment les bêtes carnivores et herbivores et les hommes ${ }^{69}$, sera freiné par l'action conjuguée des deux castrats - Tryphon et le Bœuf. Le sevrage définitif - pour une période de quarante jours, aura lieu le jour du carnaval du Lundi pur.

Quarante jours après le carnaval, ou bien cinquante jours (sept hebdomades) après Tryphon Zarezan l'équinoxe de printemps coïncidera avec la pleine lune pascale du 25 mars ${ }^{70}$. Or, le 25 mars (l'Annonciation) c'est la date fixe du retour de tous les êtres naturels et surnaturels qui hibernent: les samodivi, les dragons, les serpents, les ourses... Le «mois » pascal de cinquante jours (une " pentecôte »...) se terminera à la fête de Saint-German qui gère la grêle et le tonnerre (le 12 mai). La nouvelle cinquantaine végétarienne, annoncée par la danse muette des rusalii se terminera quelques jours après par le solstice d'été, à la fête des saints Pierre et Paul - le 29 juin ${ }^{71}$.

Dans le pire des cas, notre louve mythique engendrera soixante-huit louveteaux. La fête des kukeri aura lieu alors quarante jours après la Saint-Tryphon, c'est aux quarante martyrs du 9 mars $^{72}$, et les Pâques coïncideront avec la fête de saint Georges - patron des bergers et tueur des dragons. Or, à la Saint-Georges du 23 avril a lieu un phénomène astronomique d'une importance capitale pour le calendrier populaire : la conjonction 
des Pléiades avec le soleil ${ }^{73}$. Ce jour-là la Poussinière (Pléiades) s'éclipse pour couver pendant une période de quarante jours.

L'équinoxe du printemps et la conjonction des Pléiades avec le soleil sont donc les deux dates limites des Pâques. Ces deux bornes fixes d'essence solaire et virile, doivent être accordées avec la pleine lune du printemps. Ainsi, l'avènement des Pâques dépend en dernier lieu du «bon vouloir» de la lune fugitive ${ }^{74}$. Et si la pleine lune est à l'heure à son rendez-vous annuel avec le soleil le 25 mars, c'est une bonne année avec un printemps précoce à souhait. Sa période hivernale débutera à la coupure du 2 février pour se terminer juste au point vernal. Mais si la lune survient une journée avant la date prévue, l'éclosion du printemps sera repoussée fatalement d'une lunaison idéale, soit quatre hebdomades (vingt-huit jours) (à la Saint-Georges).

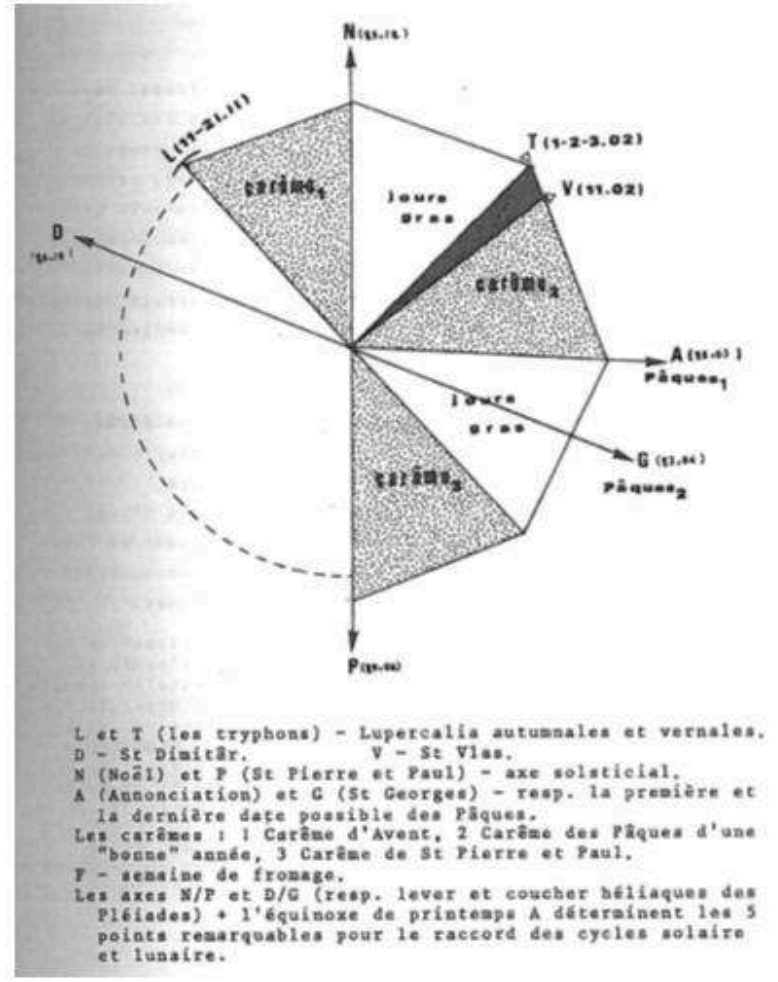

99 Personne ne songe, bien sûr, à compter des louveteaux fictifs pour retrouver la date précise du commencement du printemps. Il suffit de repérer la date décroissante de la lune en février.

Le début de la phase utérine du printemps, nous l'avons vu, est marquée par la montée de la sève des vignes. Or, si la vieille lune est, en principe, néfaste pour les travaux agricoles, c'est la seule période, dit-on, propice à la taille des vignes (Kovačev, 1914). Il s'ensuit que l'ascension du suc végétal programmée pour le $1^{\text {er }}$ février est en rapport avec la dernière phase de la lune hivernale ${ }^{75}$. Le pire de tous ces jours de déclin serait bien sûr le jour où la lune s'éclipse totalement, c'est-à-dire le jour dédié à la peste, le 10 février.

La lune croissante en Bulgarie est considérée comme propice à la majorité des travaux agricoles, ainsi qu'à l'accouchement - en somme à toutes les entreprises ou actes voués au devenir. C'est pourquoi il est souhaitable que Noël échoie en nouvelle lune. Quant au carnaval, il est indispensable qu'il soit célébré en nouvelle lune, quarante jours avant la pleine lune de Pâques. 

animale. pas aux vaches...

Le rythme des croissances, des culminations et des déclins de la lune-maîtresse du processus biologique de reproduction, est en accord avec le cycle menstruel des femmes (28-29 jours). Dans ces conditions la pleine lune serait associée à la période de l'ovulation (cf. les œufs de Pâques) et les jours sans lune correspondraient à l'écoulement périodique, impur et dangereux, du sang menstruel.

La phase ascendante de la formation de l'ovule lunaire, ainsi que la période de sa dégradation, sont évidemment infécondes: la science populaire n'ignore pas le problème du contrôle des naissances ${ }^{76}$. Et si les civilisations européennes insistent avec tant d'entêtement pour célébrer le carnaval, carnivore ou végétarien, toujours en nouvelle lune, c'est parce que cette fête agraire marque la rupture avec la reproduction

Dire que la parodie du mariage jouée par les kukeri est une hiérogamie, c'est donc sousestimer les connaissances populaires en matière végétale, ce qui est absurde pour une civilisation agricole. Les paysans bulgares, comme tous les paysans du monde, font une distinction radicale entre la reproduction hétérosexuelle des animaux et des hommes et la génération parthénogénétique (monosexuelle) des plantes. Les paysans ne s'y trompent pas lorsqu'ils affirment à travers le mythe et le rite, que la castration est une action fructifiante pour les vignes, et ne l'est pas du tout pour les femmes. De même, ce qui est utile pour la culture du blé (la castration du taureau notamment), ne convient

Mais si les divinités émasculées parviennent à neutraliser pour un certain temps l'hétéronomie animale, faut-il encore arracher les plantes nourricières de la maitrise sauvage des femmes ? Faut-il, donc, dénaturer la génération végétale "instinctuelle » et dépourvue de finalité, pour la transformer en production planifiée et organisée ?

Une fois de plus après l'invention du vin, il s'agirait de suppléer au processus naturel (féminin) par une technique pseudo-féminine - d'où la ruse du travestissement lors du carnaval. Ainsi, les seules personnes avec lesquelles les kukeri simulent le coït, travesties ou non, sont des hommes. À l'égard des femmes ils observent une attitude nuancée: respectueux ou indifférents envers les dames, ils poursuivent impétueusement les demoiselles. Cet acharnement contre les jeunes filles-épouses potentielles, ne pourrait être interprété comme désir sexuel «positif» stimulant la fécondité : les vierges persécutées se dérobent pour de bon.

Le mutisme obstiné des kukeri fait partie de la même révolte "misogyne » : la parole, pour autant qu'elle soit performative, est l'apanage des femmes. Ni le mot, ni la fonction sorcière (veštica) n'a d'équivalent masculin. Et les bardes de la tradition orale bulgare - chanteuses et conteuses - sont principalement des femmes.

Emportés par le délire iconoclaste, les jeunes travestis n'hésitent pas, sous les traits de la vieille kukerica à bafouer la cheftaine de tous les saints, en personne : Vendredi ${ }^{77}$. En effet, les fonctions sacrées de la Grande Fileuse, protectrice des liens conjugaux et de la fécondité féminine, sont tournées en dérision tout au long du spectacle de carnaval. Cette attitude blasphématoire, comme tous les gestes de la dissidence « misogyne », est bien sûr programmée d'avance et elle "coïncide " avec la clôture temporaire de la saison du tissage et des mariages, inaugurée par la fête de Sainte- Vendredi le 14 octobre. Ainsi, la prohibition bizarre, sinon irrationnelle sur le plan technicoéconomique du filage, du tissage et de la couture pendant les fêtes des loups et les carêmes $^{78}$ se justifie parfaitement lorsque l'on se souvient que les techniques 
textiles échappent à la « récupération » masculine. Il est donc normal que ces travaux de femmes soient interdits pendant les périodes qui réactualisent la grande révolution technologique : le passage de l'agriculture « douce » à la houe, à l'agriculture intensive à la charrue. Dès lors, l'assimilation courante du labourage à l'acte sexuel se trouve infirmée. Certes, la charrue est un symbole phallique. Cependant ce phallus est un phallus dévitalisé (coupé) ${ }^{79}:$ il n'est plus un organe de reproduction biologique, mais un instrument, assurant la sublimation de l'énergie sexuelle en force de travail.

La cérémonie nuptiale parodiée par le carnaval apparaît donc comme une contestation radicale non seulement de l'institution matrimoniale, mais aussi comme un refus intégral de tout rapport hétérosexuel. Toutefois, le simulacre d'ensauvagement auquel se livrent les jeunes gens en rupture avec la bonne conduite sociale ne se borne pas à commémorer et à recréer l'avènement annuel de l'agriculture. Pris dans le jeu, les hommes masqués transcendent le passé-présent-futur du temps réel, pour instaurer en l'espace d'une journée le monde surréel de la spiritualité immaculée... Le temps du Carnaval n'existe pas...

110 Au-delà et en deçà des aspects cosmologiques et agraires le carnaval bulgare exprime, bien sûr, la contestation politique d'un peuple privé de parole qui a subi cinq siècles de colonisation ottomane et presque mille ans de domination religieuse de l'Église byzantine ${ }^{80}$.

\title{
AU COMMENCEMENT ÉTAIT LE gESTE...
}

\author{
Filer ou ne pas filer \\ Tisser ou ne pas tisser \\ Jeûner ou ne pas jeûner \\ Faire l'amour ou s'en abstenir \\ Couper ou ne pas couper \\ Parler ou se taire
}

Faire ou ne pas faire...

111 Ces deux séries complémentaires d'actions prescrites ou prohibées règlent le comportement culturel d'un groupe humain au cours des périodes bien définies du cycle annuel. Elles sont exprimées par les deux «coups de serpe » de Tryphon: l'un «positif », l'autre « négatif ».

112 La position-clé dans le mythe de ce couple de gestes laconiques («comme ça » (+) et «non pas comme ça » (-) confère au récit un statut marginal par rapport aux « vrais » textes. En effet, dans un texte «normal » les gestes sont subordonnés à l'énonciation verbale qu'ils accentuent, concrètement, complètent ou commentent (Calame-Griaule, 1977).

Dans notre texte, ce « rapport de force » se trouve subverti. Il en résulte que ce mythe ne pourrait pas se traiter comme un système autonome et fermé, car ses relations avec les actes symboliques et les gestes rituels qu'il suggère et commente ne sont pas toujours du type signifiant / signifié.

114 Une explication de texte plus ou moins orthodoxe - découpage en séquences, analyse des éléments discursifs, des actes, de situations, des personnages et des objets symboliques par des plongées fictives dans le contexte social - pourrait, bien sûr, éclairer certains aspects du mythe. Mais la polyphonie aphone du geste de coupure ne se 
laisse pas appréhender par une telle méthode d'analyse. Par la force des choses donc, dans cette étude, partie d'un récit bâtard à mi-chemin entre un texte "normal » et une expression gestuelle, j'ai dû me lancer dans le chassé-croisé des pratiques rituelles de la tryphonie, sans oublier les discours disparates qui s'y référent d'une façon directe ou indirecte. De fil en aiguille à travers le fouillis de coutumes, de croyances, de gestes et de ouï-dire émergeait une image saisissante. Cette image panoramique des sacres du printemps à venir, aux contours encore flous en l'état actuel de la recherche, est orchestrée par les deux castrats divins : Tryphon et Volos.

Au terme de cette analyse " éclatée » on pourrait donc conclure que le récit de Tryphon et le rite des kukeri ne font qu'un seul mythe parlé, mais surtout joué. Les mots du texte et les gestes du carnaval muet de ce dromenon ${ }^{81}$ populaire s'interpellent et s'expliquent mutuellement sans jamais fusionner, et pour cause !

Un tel acte incestueux ruinerait à jamais la révolution mythique déclenchée par le geste du Coupeur-coupé.

\section{BIBLIOGRAPHIE}

ARISTOTE, 1969, Histoire des animaux, Paris, Les Belles Lettres.

ARNAOUdOV, M..$^{82}$, 1943, Očerki po bălgarskija folklor, Sofia, Ministerstvo na rarodnoto prosvestenie, 659 p. / « Obicai pri razdane », pp. 606-615.

ARNAOUdov, M. 1972, Studii varxu bǎlgarskite obrjadi i legendi, t. II, BAN, Sofia. / Livre II : « Kukeri i rusalii », pp. 7-218 ( $2^{\mathrm{e}}$ ed.).

CAlAME-Griaule, Geneviève, 1977, Pour une étude des gestes narratifs, in Geneviève CalameGriaule (éd.), Langage et cultures africaines, Paris, Maspero.

ČEPENKOV, M., 1900, Prikaski za čerkovni lica i javlenija ot Prilep, SbNu, 16-17, pp. 295-296.

ČERKEZOVA, M., Manuscrit, « Le Carnaval des kukeri en Bulgarie », Objets et Mondes.

DERŽAVIN, N. S., 1914, Bolgarskie kolonii v Rosii (Tavričeskaja, Xersonskaja i Besarabskaja

gubernii). Materialy po slavjanskoj etnografii, $S b N u, 29$.

DetienNe, M., 1977, Dionyos mis à mort, Paris, Gallimard.

DouRnES, J., 1975, La Toupie incestueuse, ou De la Distinction nécessaire, L'Homme, XV (3-4), p. 31-53.

GABJOV, P., 1900, Narodni kalendari, SbNu, p. 16-17 ; 19-24.

GAIGNEBEt, C., 1974, Le Carnaval, Paris, Payot.

HIPPocrate, 1970, De la génération. De la nature de l'enfant, Des maladies, Du Fœtus de huit mois, t. XI, Paris, Les Belles Lettres.

JolLy, R., 1970, Notice, in Hippocrate, t. XI, Paris, Les Belles Lettres.

KonstANTINOV, X., 1896, Običai prez godinata, $S b N u, 123$, p. 19-13. 
KovACEV, J., 1914, Narodna astronomija i metereologija, SbNu, 30, p. 1-85.

KRAJCANEC, I., 1891, Prikazki za čerkovni lica i javlenija ot Veles, SbNu, 6, p. 122.

LÉVI-STRAuSs, Claude, 1968, Origines des manières de table, Paris, Plon.

MARINOV, D., 1891, Ziva starina, I, Ruse.

MARINOV, D., 1892, Ziva starina, III, Ruse

MARINOV, D., 1914, Narodna vjara i religiozni običai, $S b N u, 28$.

MEgAS, G., 1958, Greek Calendar Customs, Athens, Press and Information Department, Prime

Minister's Office.

NonNos, 1976, Les Dionysiaques, Paris, Les Belles Lettres, t. I, II.

OPPIEN, Cynegetica, cité d'après Detienne, 96.

Отто, W. F., 1969, Dionysos. Le Mythe et le culte, Paris, Mercure de France.

Peneva-SABEVA, L., 1971, Tradicionno Lozarstvo i vinarstvo v Petričko i Menilniško, IEIM, 13, pp. 53-93.

PLINE l'ANCIEN, Histoire naturelle, Livres II, VIII, XIV, XVIII, Paris, Les Belles Lettres.

Romaios, S. A., 1949, Cultes populaires de la Thrace, Athènes, Institut Français d'Athènes.

RouBIN, L., 1971, Les vignerons danseurs de la Saint Tryphon, Objets et Mondes, XI, (2).

SLAVEJKov, P. R., 1895, Djado Vǎčar, Den, I.

SlaVejKov, P. R., 1896, Prikazki za cerkovni lica ot Tarnovsko, SbNu, 13, pp. 183-187.

SLAVEjKov, P. R., 1924, Balgarski narodni običai i vjarvanija, Sofia.

SPOROSTRANOV, 1900, Narodni kalendari ot Oxrid, SbNu, pp. 16-17.

TANOVIC, S., 1939, Srpski narodni običai u Gevgelijsko kazi, Srpski Etnografski zbornik, t. 54, Belgrade.

THÉOPHRASTE, Causes des plantes, cité d'après Detienne, 94.

VAKARELSKI, Xr., 1974, Etnografija na Balgarija, Sofia, Nauka i izkustvo.

XAJTOV, N., 1958, Selo Javorovo, Asenovgradsko, Sofia.

ZVEginCev, V. A., 1968, Teoreticeskaja i prikladnaja lingvistika, Moscou, Prosvescenie.

Abréviations

BAN - Balgarska Akamdemija na Naukite (Académie bulgare des Sciences), Sofia.

IEIM - Izvestija na Etnografskija Institut i Muzej (Bulletin de l'Institut d'ethnographie et Musée), Sofia.

SbNu - Sbornik za narodni umo-tvorenia (Bulletin du folklore bulgare), Sofia.

\section{NOTES}

1. Les usagers ne sont pas conscients de l'ambiguïté du nom qui n'a aucun sens en bulgare.

2. $1^{\text {er }}$ février de l'ancien style, c'est-à-dire du calendrier julien et 14 février du nouveau style ou calendrier grégorien. Le dernier calendrier, officiellement adopté en 1916, n'est pratiqué 
réellement que depuis une trentaine d'années, et encore... On utilise ici de préférence la notation chronologique de l'ancien style qui est plus fidèle aux prescriptions de la liturgie populaire.

3. Cette suie est récoltée la veille de Noël en balayant la cheminée. Mélangée avec des noix et mise dans un mouchoir, elle est conservée précieusement pour la tryphonisation des vignes.

4. C'est un arbre non fruitier, donc mâle. Il arrive que l'on choisisse (Marinov, 1914, 325) pour arbre sacré un noyer ou un poirier sauvages, mais jamais un arbre cultivé - quel que soit son «sexe ». L'arbre, avec la pierre sacrificielle en dessous, ainsi que la croix plantée derrière la pierre représentent l'autel du sacrifice collectif. Pour les rites de sacrifices domestiques, présidés souvent par des femmes, c'est le foyer ou le seuil de la maison qui tient lieu d'autel.

5. Selon Marinov $(1914,249)$, on égorge un bovin lorsque l'assistance est trop nombreuse. Ainsi, « on ne fait jamais rôtir (pour un sacrifice) un taureau, ni une vache stérile, mais on pourrait rôtir un veau ".

6. L'intégrité de l'animal est de règle pour le rôti sacrificiel.

7. ... pour qu'ils ne soient pas piétinés par les hommes ou bien mangés par les chiens, selon Marinov $(1914,256)$.

8. Pour la description de la Saint-Tryphon, voir Marinov (1914, 109, 356-360), Slavejkov (1924, 1895, 1896), Arnaoudov (1972, 114-127), Peneva-Sabeva (1971), Xajtov (1958), Roubin (1971).

9. Ce dévidoir est un bâton fourchu aux deux extrémités, d'une longueur d'environ quatre-vingt centimètres.

10. Il arrive parfois, mais ceci est évidemment exceptionnel, que l'époux lui-même soit reconnu stérile. Dans ce cas, il appellera un barbier qui en le rasant lui fera trois entailles au cou.

11. ... où l'on noue la chaîne de la cheminée, on éteint le feu, on cache les pinces, le tisonnier et le râble dans le four dont on enduit de bouse l'entrée, on met de la bouse également sur le peigne à fils, on macule la porte d'entrée de la maison, etc. Toutes ces opérations magiques ont pour but de lier la gueule des loups et de les rendre aveugles.

12. Les trois Tryphons (trifunci) des 1-2-3 ancien style/14-15-16 nouveau style février correspondent aux Lupercalia romaines célébrées le 15 février. Lors de cette fête consacrée à Faunus, les prêtres luperci après avoir immolé un bouc, couraient entièrement nus à travers la ville en frappant les femmes avec des lanières de peau de bouc.

13. L'équivalent bulgare de cette expression française serait: ză vălka govorim, a toj v kosărata (« on parle du loup, et il est dans la bergerie »).

14. Selon le proverbe grec, l'hiver dit : "J'envoie le message (mínima) pour mon arrivée à la StMênas (le 11 novembre) et j'arrive à la St-Philippe (le 15 novembre). »

15. C'est-à-dire "le Boiteux». Kuculan pratique une anthropophagie sélective: en effet, les textes de la littérature orale ne parlent presque jamais de femmes dévorées par des loups. Aussi, lorsque les épouses enfreignent les interdits de laver, tisser, tricoter, filer ou coudre pendant les fêtes des loups, ce seront leurs conjoints qui subiront les attaques des fauves.

16. Selon Aristote (1969, V, 14 et VI, 20), les louves portent de soixante à soixante-douze jours.

17. Elles trient des grains, coupent des légumes ou des fruits, etc., mais surtout elles cousent, tricotent, filent. Et lorsqu'une histoire est livrée avec tous les détails, on dira en bulgare qu'elle est racontée « de l'aiguille jusqu'au (bout du) fil » (ot igla do konec). Et si le narrateur s'embrouille dans son récit, comme en français, il « perd le fil de son histoire ». Les hommes connaissent aussi des histoires qu'ils racontent dans les bistrots, dans les moulins pendant que les meules broient le grain, dans les campements de bergers, pendant les fêtes, etc. Mais les conteuses sont beaucoup plus nombreuses que les conteurs.

18. Qui fait partie actuellement de la République Soviétique de Moldavie.

19. L'émigration vers la Bessarabie commence vers le XVIII ${ }^{\mathrm{e}}$ siècle. Les vagues successives de cette émigration correspondent aux révoltes contre l'oppression turque.

20. Pendant cette période dangereuse la femme est appelée lexusa. La coutume prévoit, bien sûr, une série de procédures destinées à protéger la mère et le bébé, surtout contre les trois navi. Les 
navi sont des esprits féminins qui infligent différentes sortes de maladies, la fièvre puerpérale entre autres.

21. Rencontre, rendez-vous en bulgare se dit srešta, en russe ustreča. Le mot sreća en serbo-croate signifie plutôt chance; sreten (bulg.), srećen (s.cr.): chanceux, heureux; nesreta (bulg.): malchance, mauvais destin ; nesretnik (bulg.) : homme poursuivi par le destin. La personne choisie pour le roi des vignes doit être obligatoirement un homme chanceux.

22. gevrek, qui est aussi un euphémisme pour « fesses ».

23. Village situé à douze kilomètres de Sofia, réputé pour ses sources thermales.

24. Il m'est arrivé à moi-même de « souhaiter la bonne fête » aux amis...

25. L'excellent article de J. Dournes sur la toupie incestueuse des Jörai (1975) m'a beaucoup aidée dans l'analyse de « l'inceste préférentiel » évoqué par le mythe bulgare.

26. po: préfixe de postériorité temporelle «après, ensuite »; sestra: sœur; im-a : suffixe de l'aspect passif.

27. po + brat (frère) + im.

28. Chaque fois que les samodivi replongent dans l'eau, elles retrouvent leur virginité.

29. Dans un mythe extrêmement répandu, le héros dérobe la tunique et les ailes d'une samodiva et l'oblige de cette façon à le suivre. Devenue son épouse, la samodiva lui donne des enfants. Mais en fin de compte, elle parvient par ruse à reprendre ses « vêtements » et s'envole.

30. «... Dis-moi la naissance de Bacchos le deux fois-né, celui que Zeus tira, humide du feu, enfant à demi formé d'une mère qui n'eut pas d'accoucheuse, celui qu'après s'être incisé la cuisse de ses mains hésitantes, le dieu porta dans une matrice mâle, à la fois père et mère vénérable : il avait déjà connu semblable parturition dans sa tête féconde, lui qui, portant jadis une étrange tumeur à son front gravide, avait fait jaillir Athéna aux armes fulgurantes » (Nonnos, 1976, I, 1).

31. Le vocable slave zem(l)ja (terre) ainsi que le nom de la déesse trako-phrygienne Zemelô (Zemina-Zeminder chez les Baltes) dérivent d'une même racine : zem / $\mathrm{zm}$.

32. Du verbe obričam : promettre de sacrifier, consacrer; d'où le substantif obrok: chose promise à être sacrifiée. Le terme usuel qui désigne le sacrifice (šertva) n'est jamais employé dans le vocabulaire rituel. En effet, le sacrifice « sacré » qui implique un contrat individuel ou collectif ordonné par la réglementation sociale du rite n'a rien à voir (ou si peu...) avec le sacrifice « profane », spontané et gratuit.

33. L'animal ainsi que le plat sacrificiel sont désignés par un même terme, d'origine turque kurban.

34. Les plats festifs sont dans la plupart des cas des rôtis. Or, le four paysan est toujours extérieur à l'habitation : soit dans la cour, soit aménagé sur une façade de la maison (Vakareslki, 1974, 307-310). Mais pour les grandes fêtes, comme celle de la Saint-Georges, « on faisait rôtir les agneaux dans un trou, ou bien dans un four commun à plusieurs maisons" spécialement construits (Marinov, 1914, 144). Même à Sofia, il y a une dizaine d'années, presque toutes les ménagères portaient leurs plats à rôtir à la boulangerie.

35. Il y a quelques années une dame bulgare en voyage touristique à Paris s'est plainte à moi d'avoir été « mal reçue » par une famille française : « Ils m'ont servi de la viande à peine cuite et des légumes bouillis tout à fait à part. La femme ne s'est vraiment pas donné la peine de faire la cuisine !»

36. Dans la maison traditionnelle balkanique il n'y a ni gril, ni broche. Ainsi pour faire griller le poulet sacrificiel (préalablement bouilli, bien sûr), les femmes le posent sur un trépied.

37. Fœtus se dit en bulgare (et en russe aussi) plod (fruit).

38. Du grec klêma : sarment.

39. Le vocable pirustija signifie trépied et prostituée.

40. C'est moi qui souligne.

41. La plus grande partie du savoir médical d'Hippocrate émane de la pratique des guérisseurs, pratique qu'il a eu le mérite de systématiser. Et si l'on peut parler dans son cas d'une démarche 
scientifique à l'époque où "toute la science grecque, à peu de chose près, se situe au même niveau préscientifique, qui se perpétue ailleurs, parfois en s'aggravant, jusqu'au $\mathrm{XVIII}^{\mathrm{e}}$ siècle » (Jolly, 1970), elle réside dans le rejet de la fabulation philosophique au profit d'un art expérimental.

42. Fermenter - du lat. fervare (bouillir) en passant par fermentare du bas latin; le terme populaire qui désigne la fermentation du vin en bulgare est kipene, kipeš : bouillonnement.

43. À ce propos, j'aimerais mentionner un rituel grotesque instauré vers le milieu du siècle dernier par un dénommé Vălcăn (de valk: loup) de la région de Pianec (Bulgarie de l'Ouest). Dans cette région digne de son nom (Pianec signifie «ville ou village des ivrognes ») à cette époque il n'y avait pas de pope. À la Saint-Tryphon ce père Vălcăn communiait les fidèles... avec la sève des sarments recueillie dans un bol! (Slavejkov, 1895).

44. Le vocable esprit dérive du latin spiritus : souffle; au sens propre il signifie alcool : cf. esprit-devin (alcool éthylique) et esprit-de-bois (alcool méthylique). L'intelligence artificielle des ordinateurs ne fait pas la différence entre sens propre et sens figuré. Ainsi, un ordinateur américain dans les années cinquante avait traduit l'expression anglaise « the spirit is willing, but the flesh is weak »... par la phrase «l'alcool tient bien, mais la viande est pourrie » (!)... (cité d'après Zvegincev, 1968, 192).

45. Le nom populaire du mois de février est Sečko : coupeur. «Le Coupeur coupe, Maria (Mars) dépèce et Avril vend les peaux », proclame le dicton. La notion de coupure est liée ici au froid hivernal qui coupe... le souffle et tue.

46. Cf. Aristote (IX, 6, 2) ; Théophraste (VI, 5, 2) ; Pline (VIII, 23, 62); Detienne (1977, 3).

47. «Le lion reconnaît à l'odeur l'adultère commis par la lionne avec le léopard... » (Pline, VIII, $17,43)$. Le vocable « léopard » lui-même résulte du «croisement » du lion avec le pard : leopardos gr.

48. Pardos gr. signifie « puanteur »: l'animal avait reçu son nom à cause de ses pets nauséabonds. 49. Sur la fonction sémantique de la panthère parfumée voir l'analyse très fine des mythes de « chasse et séduction » de M. Detienne (1977, ch. II).

50. sirni zagovezni ou sirni signifie "de fromage » et le mot za-govezni est formé du préfixe za désignant un commencement + le radical du verbe goveja (jeûner) + zni - suffixe nominal archaïque.

51. À comparer avec le jeu carnavalesque français appelé «la farce de la figuette»: objet suspendu à un fil fixé au bout d'une canne à pêche.

52. pesiponedelnik - de pes (chien) et ponedelnik (lundi), équivalent au skylodeftéra grec. Le nom

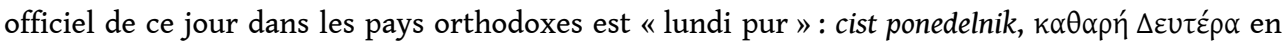
grec. Très souvent le carnaval intervient le lundi de la semaine de fromage. Mais ceci n'altère pas les principes de l'analyse proposée : le carnaval serait alors une fête modérément végétarienne.

53. Le verbe goveja (bulg.), govet' (russe), dérive du vocable govedo (« bovin ») - tchèque hovado, serbo-chr. govedo, ukr. Govjado, lit. guws (vache), grec bous, lat. bos, bovis, v. sl. govēdo; skr. gāus (taureau, vache) < i.e. ${ }^{*} g^{u}$ ous (taureau).

54. Jadis les jeunes mariées devaient «boviner » c'est-à-dire se taire devant les beaux-parents pendant la quarantaine de jours qui suit la cérémonie de mariage. Selon les textes de la littérature orale (mythes et contes), elles devaient observer cette attitude pendant plusieurs mois. Par la suite, ce mutisme rituel s'est vu réduit à quelques jours. Sous une forme plus ou moins atténuée, la coutume est conservée jusqu'à nos jours.

55. Mais il est particulièrement riche et mieux conservé en Thrace actuelle, partagée entre la Bulgarie, la Turquie et la Grèce. En effet, il n'y a pas de différences très sensibles entre le rite grec des Kalogeroi et celui des Kukeri de la Bulgarie du Sud-Est.

56. Des cornes de bœuf, de bouc, ou de cerf.

57. Le rôle de l'ours est parfois joué par un chien couvert de peau d'ours (Marinov, 1914, 377). 
58. Selon Vakarelski (1974: 606), parfois le Kuker touche les pieds des femmes stériles afin de leur procurer la fécondité. Ce geste respectueux est très différent de la fustigation des hommes et des jeunes filles, fustigation qui est un acte d'agression ou de viol. Cet attouchement rappelle plutôt l'arrosage des racines du cep avec du vin à la Saint-Tryphon. Somme toute, lors du carnaval, le comportement des kukeri envers les femmes demeure toujours chaste.

59. Dans certains villages ce phallus était propriété communale que l'on conservait soigneusement (Vakarelski, 1974, 606).

60. Le rôle du roi du carnaval grec à Adrinople est tenu par un personnage surnommé SeigneurChien (Köpek-bey en turc). Monté sur son char, un panier troué décoré de têtes d'ail et d'oignons en guise de couronne, Sa majesté dirige en personne la poursuite des chiens le Lundi pur (Romaïos, 1949, 145).

61. La première semaine du carême est appelée « semaine de la rage » ou " semaine de la folie ».

62. Certaines troupes n'ont pas de roi. Dans ce cas, le kuker cumule la fonction de chef religieux avec celle de souverain. Il arrive aussi que tout le pouvoir soit concentré dans les mains de la Vieille (Marinov, 1914, 373-377).

63. Pour la description détaillée des variantes, voir Marinov (1914, 373-378) ; Arnaoudov (1972, « Kukeri i rusalii »); Vakarelski (1974, 606, 699-710); Čerkezova (manuscrit); Romaïos (1949).

64. C'est moi qui souligne.

65. «Nos rituels (...) ont peu d'originalité ; dans la plupart des cas il s'agit soit de rites et fêtes antiques modifiés, soit de simples emprunts " (Arnaoudov, 1972, 214).

66. Attesté dans les textes médiévaux depuis le XII ${ }^{\mathrm{e}}$ siècle.

67. Rosa: «rosée»; rosen, -a, -i, est aussi un adjectif («couvert de rosée »). Le dictame effectivement a les propriétés d'un médicament calmant. Mais c'est aussi une plante vénéneuse.

68. Dans toutes les langues slaves vol signifie «bœuf» - cf. lat. bos. Le nom grec de saint Vlas, $B \lambda \alpha ́ \sigma i o$, a donné Blaise en français. La fête de Saint-Blaise en Europe Occidentale est le 3 février et la semaine des laitages est partiellement absorbée par le carnaval gras : cf. les crêpes de Mardi gras. Ayant fait l'économie de la période transitoire, les Occidentaux se sont trouvés dans l'obligation de charger saint Blaise de la double fonction de protecteur des loups et des bœufs.

69. Chez les Russes et les Ukrainiens le 5 février - Sainte-Agathe la Vachère (Agafja-korovnica) est la fête de la peste.

70. La date de l'équinoxe de printemps pour la tradition populaire est fixée au 25 mars.

71. Cette cinquantaine est formée par le jeûne qui précède la Fête-Dieu auquel s'ajoute celui des deux saints. La durée prescrite du jeûne des saints Pierre et Paul est d'un mois ; il y aurait donc une rupture de quelques jours entre les deux jeûnes.

72. Selon la croyance européenne, les trois derniers jours de mars sont empruntés à février. On les appelle « les jours comptés » en bulgare et « jours de la vache » en langue d'oc. Pour obtenir le bon compte il faut donc les rendre provisoirement au mois de février.

73. Il y a deux mille ans l'équinoxe du printemps avait lieu le 25 mars, donc exactement à la date assignée par le calendrier populaire bulgare. À une époque très reculée, d'où nous vient probablement le culte des Pléiades, l'équinoxe de printemps avait lieu le 23 avril. Le recul graduel est dû au phénomène de précession.

74. Le sexe de la lune en bulgare est flottant : elle est appelée tantôt Sieur Lune, frère cadet du Soleil (mesec), tantôt Dame Lune (mesečina).

75. Ce calcul lunaire est en désaccord avec l'affirmation de C. Gaignebet selon laquelle le 2 février de l'année antérieure (ici la «bonne » année) échoit en nouvelle lune, ce qui entraîne une pleine lune en Noël. Ce comput faussé - et l'auteur en est conscient, trouve une certaine justification dans l'interprétation qu'il m'avait suggérée au cours d'une conversation : le temps des carnavals (celui des douze jours après Noël ou la fête des fous, et le Mardi gras) serait un temps inversé qui marche à reculons. On pourrait y ajouter une inversion de $180^{\circ}$ de l'orientation des cornes de la vieille lune réglementaire qui va la rajeunir nécessairement. Pour la même 
occasion, le carnaval avalera sous forme de crêpes lunaires les dix-onze jours qui gênent la bonne marche des événements.

76. Un enfant conçu le jour même de l'ouverture de la saison des mariages - le 14 octobre, sera mis au monde dix lunaisons plus tard à la Saint-Élie du 20 juillet, qui est une très grande fête dans les pays " orthodoxes "; celui qui serait programmé à naître à la Sainte-Vendredi la fileuse (Sveta Petka) du 14 octobre, devrait être conçu à la fête des sages-femmes, le 8 janvier. Quant au jeûne sexuel du carême de Pâques, il évite tout simplement les naissances en carême automnal. On pourrait facilement multiplier les exemples.

77. Paraskov'ja-pjatnica (Parascève-vendredi) ou Paraskov'ja-L'njannica chez les Russes ; Petka en bulg. - du pet (cinq) et petak (cinquième jour de la semaine) ; $\pi \alpha \rho \alpha \sigma \kappa \varepsilon v ́ \alpha \sigma \mu \alpha$ gr. " préparation ». La déesse balkanique et slave Vendredi n'a rien à voir avec la Vénus-Aphrodite-Vendredi de la semaine occidentale. S'il fallait lui chercher des parèdres dans le panthéon emblématique de l'antiquité, elle serait à comparer à Athéna et à sa concurrente malchanceuse en matière de tissage, Arachnée. La pelote de fil conducteur nous fait penser aussi à Ariane, d'autant plus que cette dame, délaissée par Thésée, avait épousé en secondes noces Dionysos.

78. La prohibition est impérative pendant les fêtes des loups et les temps « forts » du carême : le début et la fin. Par ailleurs, tout le long de l'année, il est interdit de filer, tisser et coudre le vendredi. Cette prohibition des travaux textiles pendant le carnaval du Mardi gras et au début du carême est observée partout en Europe.

79. La charrue est tirée par des bœufs qui sont des taureaux châtrés, et dans le rite des kukeri la fonction des bœufs est remplie par des jeunes travestis.

80. Pendant le joug turc les Bulgares n'avaient pas d'Église autonome. Le gouvernement turc avait confié aux Grecs la direction des affaires religieuses des «infidèles " à confession orthodoxe. C'est pourquoi le mouvement organisé de libération de l'oppression turque débuta par une guerre religieuse contre l'hégémonie de l'Église grecque.

81. dromenon : cérémonie cultuelle dramatisée.

82. Pour éviter la prononciation incorrecte du nom d'Arnaoudov, cité à plusieurs reprises dans le texte, il est écrit selon les normes de l'orthographe française.

\section{RÉSUMÉS}

L'étude de ce mythe ou « légende » étiologique est associée au rite vernal de la taille des vignes en Bulgarie. Tryphon se coupe le nez en voulant montrer son habileté technique. Ce geste inversé, s'il est fécondant pour les ceps, apparaît aussi comme punition d'un désir incestueux pour sa sœur, la Vierge. L'analyse du texte se place dans le contexte du calendrier des diverses fêtes de printemps, en s'appuyant sur de nombreuses références à la Grèce antique. L'auteur met en valeur quelques découvertes, en apparence paradoxales, mais recouvrant une logique inversée : la Vierge patronne des loups et protectrice des louves en couches, le rôti peut être bouilli, les rites de Carnaval sont muets, l'acte de fécondité agraire est mené par un castrat: le bœuf qui renvoie à Tryphon, l'autre castrat. Enfin, est mise en relief l'importance du geste symbolique, orchestrant les divers aspects du jeûne pascal, à la fois alimentaire, verbal et sexuel.

This myth or etiological "legend" is associated with the Spring rite surrounding the pruning of vines in Bulgaria. Tryphon cuts his nose as he tries to demonstrate his technical know-how. This inverted gesture, which seems to be fecund for the vine stock, can also be seen as a form of 
punishment for his incestuous desire for his sister, the Virgin. This analysis places the text within the context of various Spring-time festivities based on numerous references to Greek antiquity. The author foregrounds several discoveries that seem paradoxical but that correspond to an inverted logic: the Virgin is the patron of the wolves and protects birth-giving female wolves, the roast can be boiled, the rites of Carnival are silent, the act of agrarian fertility is led by a castrated being: the ox refers to Tryphon, the other castrated being. Symbolic gesture is highlighted, orchestrating different facets of the Easter fast, be they verbal, sexual, or foodrelated.

\section{INDEX}

Thèmes : anthropologie (Europe)

Mots-clés : autocastration, taille de la vigne, fêtes calendaires de printemps, rites agraires, fécondité, gestuelle performative, jeûne pascal

Index géographique : Bulgarie

Keywords : Bulgaria, Autocastration, Pruning Vines, Spring Calendar Feasts, Agrarian Rites, Fecundity, Performative Gesture, Easter Fasting, Anthropology 